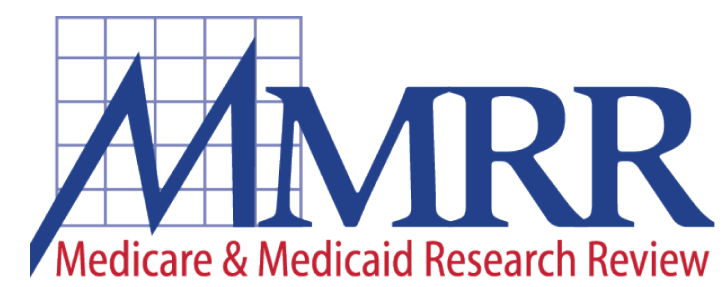

2013: Volume 3, Number 4

A publication of the Centers for Medicare \& Medicaid Services, Office of Information Products \& Data Analytics

\title{
Accounting for Unobservable Exposure Time Bias When Using Medicare Prescription Drug Data
}

\author{
Elizabeth A. Cook, ${ }^{1}$ Kathleen M. Schneider, ${ }^{2}$ Elizabeth Chrischilles, ${ }^{3}$ John M. Brooks ${ }^{1}$ \\ ${ }^{1}$ University of Iowa College of Pharmacy-Department of Pharmacy Practice and Science \\ ${ }^{2}$ Buccaneer-A General Dynamics Company \\ ${ }^{3}$ University of Iowa College of Public Health—Department of Epidemiology
}

Objective: To describe the prevalence and correlates of unobservable medication exposure time, and to recommend approaches for minimizing bias, in studies using Medicare Part D data.

Sample: 179,065 Medicare patients hospitalized for an AMI in 2007 or 2008.

Methods: We compared two methods for creating medication exposure observation periods using acute care discharge vs. post-acute care discharge dates. We examined options for increasing cohort sizes by requiring different thresholds for observable days, or by using as a covariate, in the observation period. We calculated the extent and health status correlates of unobserved Medicare Part D exposure time and examined its association with receipt of beta-blockers.

Results: $39 \%$ of patients had unobservable time during the 30 day exposure assessment period following acute care; they were significantly older, had more comorbidity and longer acute care stays, had worse 1-year survival, and were significantly less likely to be classified as beta-blocker users.
Using the alternative exposure assessment window, only $29 \%$ of the sample had unobservable time, and differences between groups were less pronounced. Significant gains in sample size can be obtained by restricting or controlling for the number of observable days required in the exposure assessment period.

Conclusions: Unobservable exposure time is common among Medicare Part D beneficiaries, and they are often in worse health. To retain patients with unobservable exposure time, we recommend stratifying patients on receipt of post-acute facility-based care, calculating and using observable days as a covariate and, when appropriate, using the discharge date from contiguous post-acute facility care for beginning the exposure assessment period.

Keywords: Administrative Data Uses, Epidemiology, Medicare, Pharmacy

ISSN: 2159-0354

doi: http://dx.doi.org/10.5600/mmrr.003.04.a01 


\section{Medicare \& Medicaid Research Review} 2013: Volume 3, Number 4

\section{Mission Statement}

Medicare \& Medicaid Research Review is a peerreviewed, online journal reporting data and research that informs current and future directions of the Medicare, Medicaid, and Children's Health Insurance programs. The journal seeks to examine and evaluate health care coverage, quality and access to care for beneficiaries, and payment for health services.

\section{http://www.cms.gov/MMRR/}

\section{U.S. Department of Health \& Human Services Kathleen Sebelius Secretary}

\section{Centers for Medicare \& Medicaid Services Marilyn Tavenner Administrator}

Editor-in-Chief

David M. Bott, Ph.D.

The complete list of Editorial Staff and Editorial Board members may be found on the MMRR Web site (click link): $\underline{\text { MMRR Editorial Staff Page }}$

Contact: $\underline{\text { mmrr-editors@cms.hhs.gov }}$

Published by the Centers for Medicare \& Medicaid Services.

All material in the Medicare \& Medicaid Research Review is in the public domain and may be duplicated without permission. Citation to source is requested.

\section{Introduction}

One of the great strengths of comparative effectiveness research (CER) lies in the evaluation of health care interventions to determine which work best for patients in real world settings (Sox \& Greenfield, 2009). To this end, CER has often relied on the use of large population-based secondary data sources, such as health care claims databases (Motheral et al., 2003). Since this type of information is generally collected for non-research purposes, great care must be taken in study design, sample selection, measurement, and control of confounders to ensure the quality of causal inferences that can be made from this research (Schneeweiss, 2007; Ray, 2003; Stukel et al., 2007).

The Medicare Prescription Drug, Improvement, and Modernization Act of 2003 created a prescription drug benefit, called Medicare Part $\mathrm{D}$, which was made available to beneficiaries in 2006. Using Medicare Part D prescription drug event (PDE) data presents both new opportunities and methodological challenges for health services researchers. Part D covers most prescription drugs; however, it excludes drugs that are covered under Medicare Parts A or B. As a result, medications taken by the patient while in institutional care settings (covered in the per diem payments under Medicare Part A) will not be observed in Part D data (U.S. DHHS, 2009). Suissa (2008) has explained the importance of accounting for hospitalized days, which he refers to as "immeasurable" when using administrative claims to measure medication exposure. Failure to consider this unobserved exposure time has the potential to misclassify exposed person-time as unexposed.

Studies of effectiveness of treatments for a particular disease commonly begin with a diagnosis date, and an examination of a specified 
period of time after a diagnosis for the purpose of classifying treatment. This paper reviews recent literature to determine the extent to which unobservable medication exposure time may be a common source of bias in studies using pharmacy claims data, and describes methodological adjustments that have been examined to date. We empirically test various approaches to identifying and addressing unobservable exposure time by exploring the extent, correlates, and impact of this issue as it relates to beta-blocker use for a sample of Medicare patients: those with the Part $D$ prescription drug benefit who were hospitalized for an acute myocardial infarction (AMI) in 2007 or 2008. Finally, we suggest potential approaches for minimizing unobservable exposure time bias when using Medicare Part D data..

\section{Background}

Ascertaining an accurate measurement of medication exposure or treatment is a critical part of designing an effective pharmacoepidemiological study. Suissa (2008) has characterized a particular type of bias he detected in several published studies that he referred to as "immeasurable time bias." Cohort or case control studies using administrative data to identify a population of subjects with chronic disease, using available diagnosis codes on physician or hospital claims, often assess drug exposure by evaluating pharmacy claims based on prescriptions usually dispensed on an outpatient basis. The time Suissa refers to as "immeasurable" is a period of time during the follow-up observation period when a subject cannot be properly identified as exposed, due either to inpatient hospitalization or other institutional care. For example, inpatient hospitalization often immediately precedes death, and patients receive medication in the hospital rather than filling outpatient prescriptions during this time. Even when a patient survives, those with serious chronic diseases may have a series of rehospitalizations during the exposure period; for studies focusing on medication adherence, even hospitalizations occurring during follow-up will belie the continuity of medication exposure when measured using outpatient claims (Suissa, 2008). Ultimately, the study subjects will not all have a uniform time period available to define exposure, and those with unobserved exposure time have a lower probability of filling a prescription on an outpatient basis. Since this population is also at a higher risk of death or other poor outcomes, this may lead to overestimates of the effectiveness of treatment.

Though there are studies examining medication adherence that address the issue of unobservable exposure time, by either adjusting the time period by subtracting unobserved days or by excluding these patients altogether (Andrade, Kahler, Frech, \& Chan, 2006; Caderette, Solomon, Katz, Patrick, \& Brookhart, 2011), this is by no means a universal approach. In a systematic review of the methodological characteristics of cohort studies by Nikitovic, Solomon, and Cadarette (2010), the authors found that two of nine studies explicitly stated that $100 \%$ compliance to medication regimens was assumed without considering the possibility of institutionalized days, while the remaining seven were silent on the issue. Adherence studies are particularly vulnerable to unobservable exposure time bias. A 2005 review of studies of adherence to antihypertensives using secondary data revealed that, of 20 selected studies published between 1995-2004, the majority did not describe methods for adjusting for hospitalized days in their analysis of the quality (Fitz-Simon, Bennett, \& Feely, 2005). In a 2009 paper by Yang et al. that used Medicare Part D data to measure adherence to oral hypoglycemic drugs, ACE/ARBs, and statins among diabetics, the formula they used to calculate proportion of days covered did not subtract institutionalized days from the denominator or 
use any other alternative method to account for this potential source of bias; although, to be fair, it is not always clear whether the problem is a failure to account for unobserved exposure altogether, or a failure to document methods explicitly.

Because it is now possible to examine relationships between medication use and clinical outcomes in the Medicare population, those who have comparatively little training in pharmacoepidemiology may look to methodological articles for instruction on use of pharmacy claims data. However, even these do not always provide appropriate guidance for measurement of drug exposures; a 2007 Value in Health article by Peterson et al. advises those using retrospective databases, to measure medication compliance and persistence, to follow a set of criteria agreed upon by the authors to ensure quality results. Though this article did not describe strategies to deal with Part D data specifically, nowhere in the article does the issue of accounting for unobserved exposure time appear. Though Cox et al., in a 2009 article that describes approaches to mitigate bias and confounding in treatment effect studies using secondary databases, acknowledge that a great number of opportunities for exposure misclassification exist given the multiple ways in which medications can be accessed, including during a period of hospitalization, no specific strategies are given for the novice researcher to account for this particular problem.

We found very few examples in the literature regarding how best to circumvent the bias of unobservable exposure time. Cadarette and Burden (2010) chose to subtract the number of days in hospital (including long-term care) from the denominator, in their measure of proportion of days covered, and excluded patients having 50\% or more days in hospital during the exposure ascertainment period. However, the authors did not explain in detail how they came to this latter decision and did not discuss how other institutionalized days (e.g. skilled nursing) were accounted for. Suissa (2008) tested six different approaches to try to quantify the bias of unobservable exposure time. One of these was simply requiring thirty consecutive non-hospitalized days in the exposure assessment period for subjects to remain in the study-an approach we consider in this paper as well. Suissa's second and third approaches retained all subjects in the sample, regardless of unobservable time, and experimented with various methods of weighting according to the number of measurable days. His final two approaches computed the measurable time during the 30-day exposure period to calculate either the 30-day rate of exposure or the 30-day cumulativeincidence of exposurebyobservabledays, the latter of which used the Kaplan-Meier estimator to account for varying observable windows of time. However, it is not clear whether Suissa recommends that any of these approaches be used to actually correct for bias; in addition, most are applied to an example using a case-control design rather than a cohort study, which is an objective of this paper.

\section{Methods}

This study uses a population of Medicare beneficiaries with the Part D prescription drug benefit, who were discharged from an acute hospitalization for an AMI in 2007 or 2008, to characterize a population that experiences unobserved medication exposure time. We empirically examine the potential for minimizing unobserved time exposure bias in the measurement of post-hospitalization beta blocker exposure, and demonstrate the impact of these methods on retaining complex subjects for CER.

\section{Our Methods}

\section{Study Population}

We obtained all Medicare claims files, enrollment information, and Part D prescription drug event 
data for patients hospitalized for an AMI in 2007 or 2008, applying the Chronic Condition Warehouse definition of AMI of ICD-9 codes 410.01, 410.11, $410.21,410.31,410.41,410.51,410.61,410.71$, $410.81,410.91$ in the first or second position of the relevant inpatient claim. We specified a 30-day exposure assessment period post-discharge to classify medication exposure. We restricted our sample to those who were discharged alive from acute care and lived at least 30 days post discharge, had continuous Medicare Part A and B fee-for-service enrollment 12 months (Part D for 6 months) pre-AMI and 12 months post-index AMI, or until death, and no hospice use 12 months pre-AMI and 30 days postAMI, resulting in a sample of 179,065 patients.

\section{Cohort Start Date}

Analyses using Medicare data that examine choice of drug therapy are often pegged to an index hospitalization, as it is a sentinel event that would likely require the initiation of-or modification toexisting drug therapy. Although some hospital stays only generate a single inpatient claim, it is not unusual for a hospital stay to give rise to multiple inpatient claims with overlapping dates. For instance, it is fairly common to observe transfers between critical access hospitals (CAH) and acute care hospitals, but it would be inappropriate to regard these as distinct hospitalization episodes of care for the purposes of identifying the index AMI event. In addition, it is also common to see additional facility-based care after an inpatient AMI admission; for example, patients may be transferred to a rehabilitation facility, a longterm care hospital, a skilled nursing facility (SNF), or a hospice facility immediately upon discharge from acute care, or shortly afterwards. If a patient is discharged from the initial acute hospitalization, and subsequently admitted to a SNF or other inpatient or hospice care facility, either immediately or during the treatment observation period, the medications administered during that time will be covered by the Medicare Part A benefit as part of that stay, but not explicitly documented on a claim. As a result, Medicare Part D claims will not be observed during this period of institutional care, although the patient was in fact receiving drug therapy. Analysis of the Part A claims immediately following the discharge date provides the ability to determine a "timeline" of care for the patient, which is essential to the identification of unobserved exposure time in the post-index period.

We compared two different methods for defining the duration of hospital stays (i.e, defining the discharge date from which we determined the onset of the cohort medication exposure observation period). The first method (Method 1) used only acute short-stay and critical access hospital (CAH) inpatient claims for the cohort of eligible AMI patients, to construct an acute stay-level file by combining those claims with overlapping admission and discharge days into a single distinct stay; if a claim discharge date occurred later than or on the same day as the admission date for another claim, those were combined into one stay. Claims that were separated by one or more days were considered readmissions and were not combined. Patients who received hospice care were excluded in our study of secondary prevention drugs for AMI. We then sorted patients into one of three groups: patients in Group 1 were discharged to community and observable all 30 days ( $\mathrm{n}=109,886 ; 61 \%)$; patients in Group 2 were discharged to community, but were unobservable at some point during the first 30 days $(\mathrm{n}=43,989 ; 25 \%)$; and patients in Group 3 were discharged immediately into institutional post-acute care, which could include a long term care hospital, psychiatric hospital, inpatient rehabilitation facility, skilled nursing facility, or any other facilities paid under Medicare Part A, and whose exposure was therefore immediately unobservable ( $\mathrm{n}=25,190 ; 14 \%$ ). The distinction between these groups is illustrated in Exhibit 1. 
Exhibit 1. Acute Care Discharge Date (Method 1) Treatment Definition

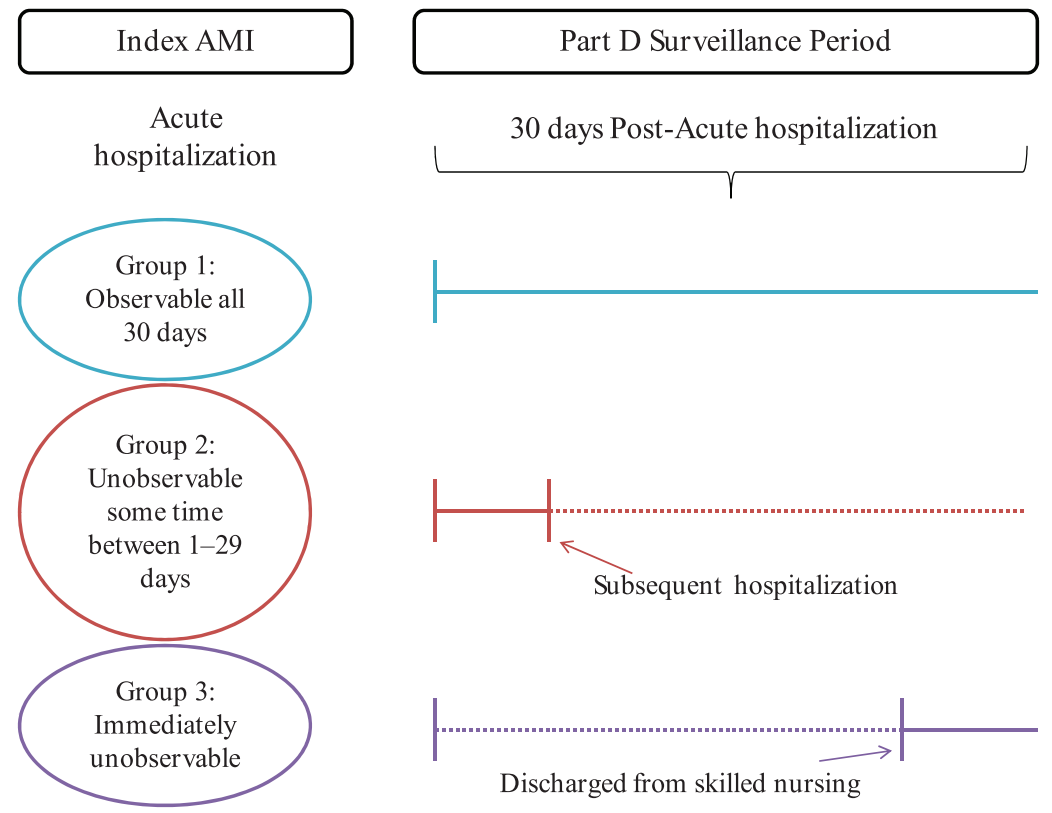

NOTE: Solid line indicates observable time; dotted line indicates unobservable time.

SOURCE: Author's illustration of methods.

We refer to these 3 groupings using the post-hospital stay time frame as "Method 1" throughout the rest of the paper.

The second type of stay-level file we constructed (Method 2) used the same algorithm as above, but rather than constructing the initial AMI stay using only acute short-stay and $\mathrm{CAH}$ claims, we considered all overlapping Medicare Part A inpatient (acute, long term care hospital, Inpatient Rehabilitation Facility [IRF], Inpatient Psychiatric Facility [IPF] and CAH) and Skilled Nursing (SNF) claims as part of the stay. The discharge date from the last claim in this "institutional" stay, which included post-acute care, was the beginning of the drug observation period for Method 2 (see Exhibit 2). We calculated total unobservable time during the first 30 days after the institutional stay discharge date.

Because a large proportion of the sample still experiences some unobservable time using either Method, we examined the impact of requiring a minimum number of observable days post-acute care discharge, and illustrate two methods for selecting a minimum exposure time frame. We also explore using the number of observable days post-acute care discharge as a covariate. Our objective was to determine the feasibility of retaining a proportion of those who are re-institutionalized at some point during the exposure assessment period.

\section{Measures}

To determine whether patients had been treated with beta blockers in the first thirty days after the index AMI stay (i.e., the exposure assessment time frame), we classified medications by merging the Medicare Prescription Drug Event (PDE) files with a resource dataset created from the Multum Lexicon Plus dataset (Copyright 2012 Lexi-Comp, Inc. and/or Cerner Multum, Inc) to obtain all prescription fill information for the drug class. Our key exposure measure involved beta blocker 
Exhibit 2. Institutional Discharge Date (Method 2) Treatment Definition

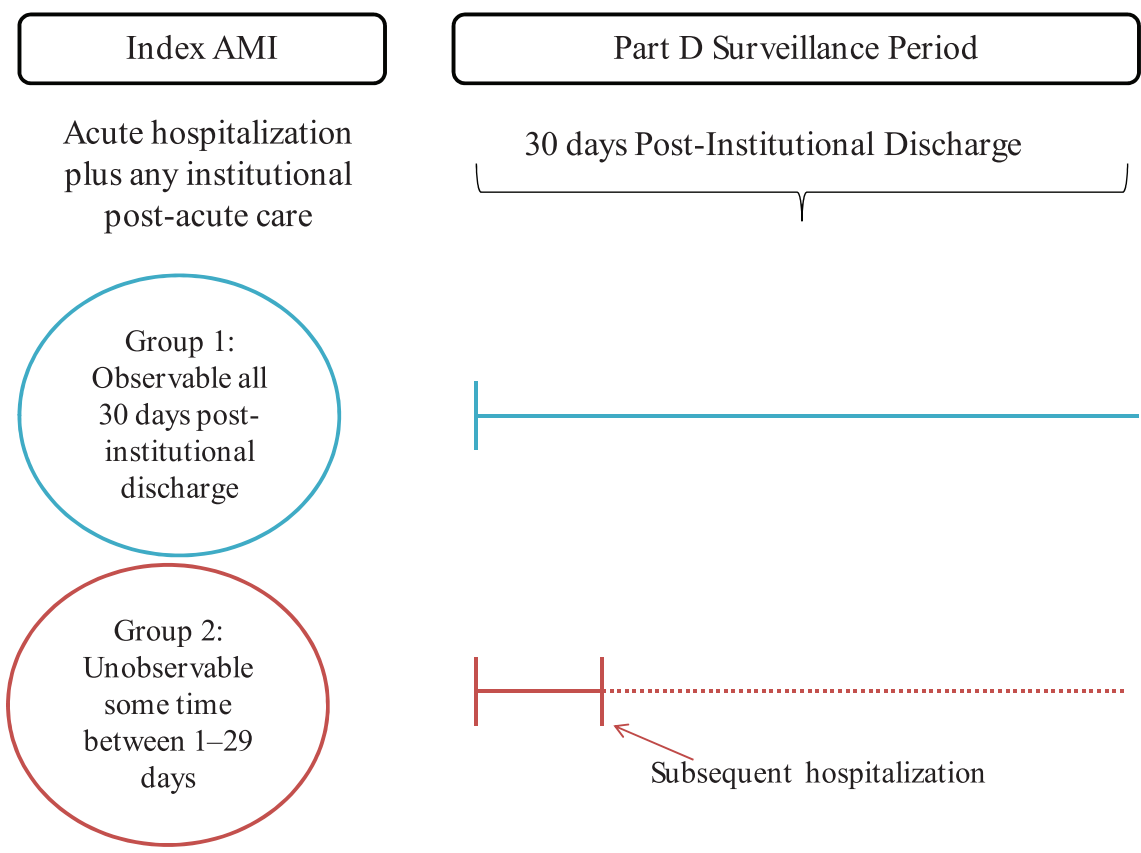

NOTE: Solid line indicates observable time; dotted line indicates unobservable time.

SOURCE: Author's illustration of methods.

treatment initiation as defined by all beta-blocker prescriptions filled within the first 30 days postindex stay.

We created five patient-level variables that were likely to be proxies for patient severity. These measures included age at acute hospital discharge; a comorbidity score as measured by the Charlson Comorbidity Index (CCI; Klabunde, Potosky, Legler, \& Warren, 2000), with a possible range of 0 to 18 , where a higher score indicates greater comorbidity; the length of the acute care hospitalization stay (LOS), in days, by subtracting the admission date from the discharge date; prior beta-blocker use ( 1 if the patient filled a prescription for beta blockers in the six months prior to admission, 0 otherwise); and 1-year survival ( 0 if the Medicare date of death occurred within 365 days of the acute care discharge, 1 otherwise). Our primary outcome measure was the initiation of beta-blocker therapy in the first 30 days post discharge ( 1 if a prescription was filled, 0 otherwise).

\section{Statistical Analysis}

To evaluate the potential impact of unobservable exposure time, we compared the two classification approaches by examining the differences in patient characteristics and in observed treatment initiation, between groups that were constructed using our acute discharge date definition (Method 1) and the groups constructed using the institutional discharge date definition (Method 2). We used analysis of variance (ANOVA) to compare means between all groups for each method on all covariates (age, CCI score, LOS, prior beta-blocker use, and 1-year survival), and applied the Bonferroni correction (Dunn, 1961) to account for the effect of multiple testing. To describe various options in thresholds for retaining patients in the sample by number of observable days, we calculated the available population, the proportion treated, and various summary statistics for each group. To determine the impact of unobservable time on the odds of being classified as treated with beta 
blockers, we performed unadjusted and adjusted logistic regressions on the odds of being observed as filling a prescription for a beta-blocker, among the subsample without pre-index beta-blocker use ( $\mathrm{n}=90,390)$, using the group definitions for both Methods 1 and 2 as explanatory variables for the first two regressions, and using the number of observable days in the 30-day window post-acute care discharge for the third regression. Patients who were observable and discharged to community all 30 days (Group 1) were used as the reference group for the first two regressions, and control variables included age, comorbidity score, and LOS. All analyses were performed using SAS 9.3.

\section{Results}

A total of 69,179 (39\%) of the sample had some unobservable time during the 30-day exposure assessment period following acute care (Exhibit 3). Using the acute care discharge date definition (Method 1), patients in Group 1-those who were discharged to community and observable all 30 days-were younger, had fewer comorbidities, were less likely to be prior users of beta-blockers, had a shorter acute care LOS, and were more likely to survive 1-year post discharge than patients in Groups 2 or 3 (Exhibit 4). All means were significantly different $(\mathrm{p}<.0001)$ between groups.

\section{Exhibit 3. Original and Redistribution of Group Membership Between Acute Care Discharge Date (Method 1) Groups and Institutional Discharge Date (Method 2) Groups}

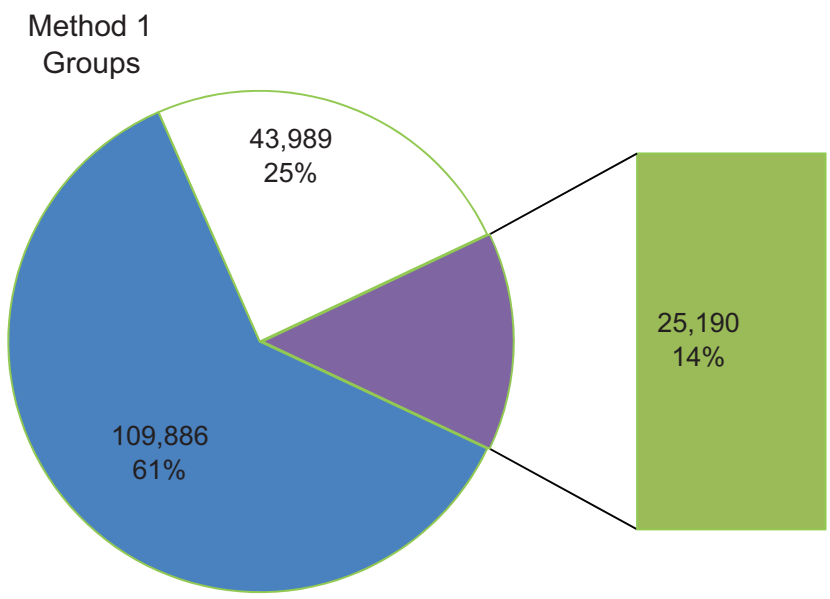

Group 1-discharged to community and observable all 30 days

$\square$ Group 2-discharged to community but were unobservable at some point during first 30 days

Group 3-discharged immediately into post-acute care

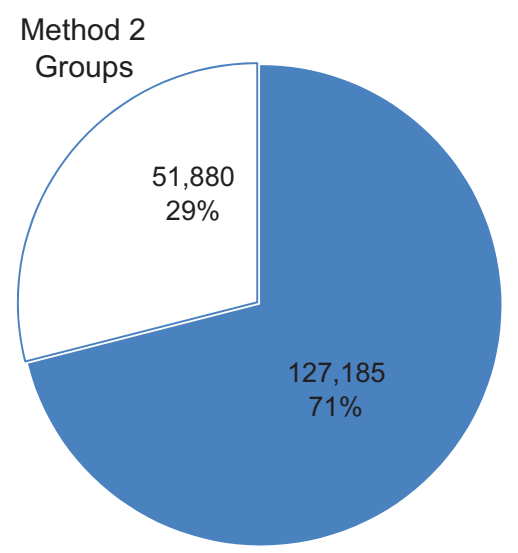

- Group 1-discharged to community and observable all 30 days (includes 17,299 from Method 1 Group 3)

$\square$ Group 2-discharged to community but were unobservable at some point during first 30 days (includes 7,891 from Method 1 Group 3)

SOURCE: Calculations based on CCW Medicare AMI cohort 2007-2008, using enrollment and Part A and B fee-for-service claims, 2006-2009. 
Exhibit 4. Frequencies and Means for Covariates for Acute Care Discharge Date (Method 1) Groups

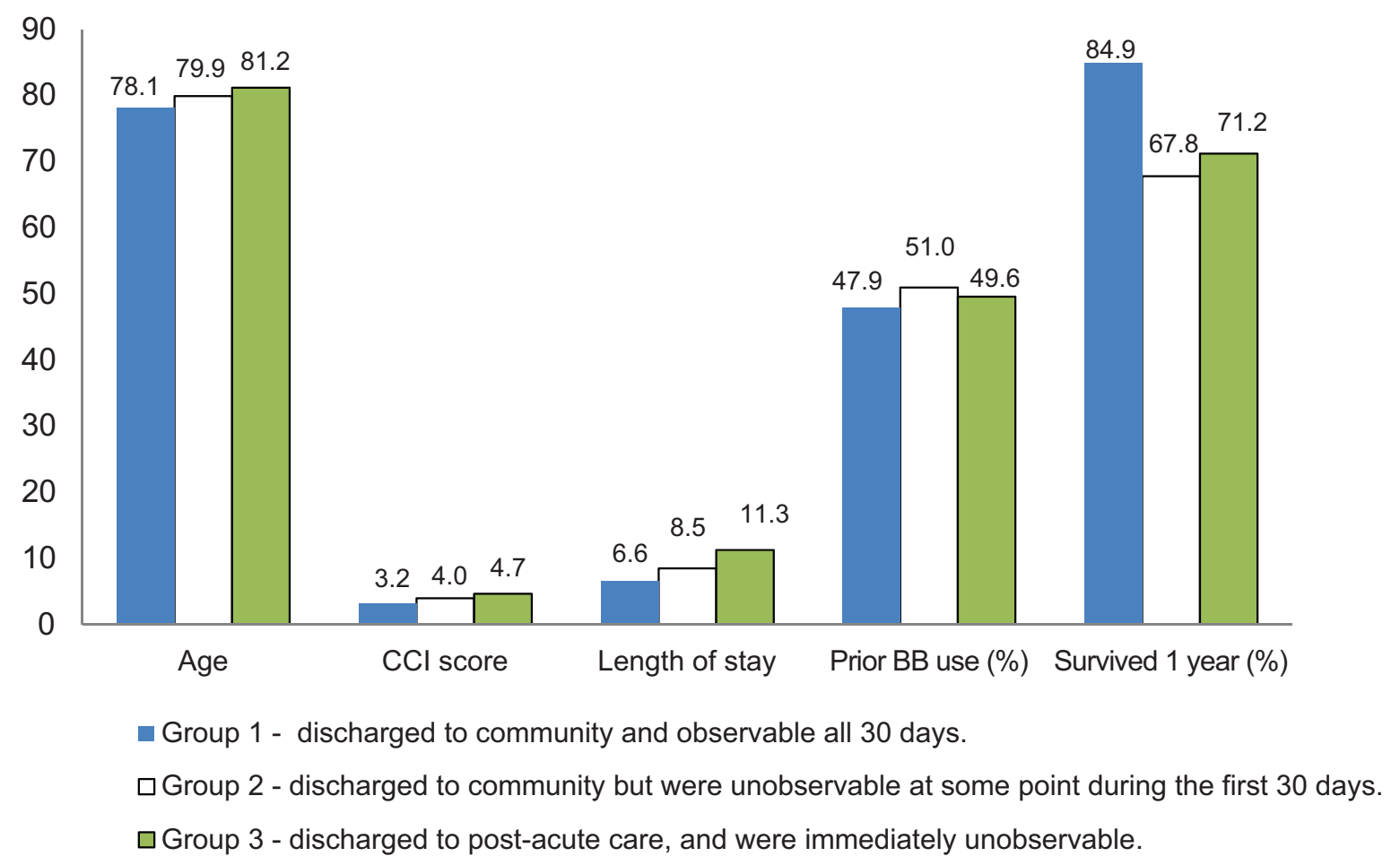

NOTE: All differences were significant at $\mathrm{p}<.0001$.

SOURCE: Calculations based on CCW Medicare AMI cohort 2007-2008, using enrollment and Part A and B fee-for-service claims, $2006-2009$.

By contrast, under Method 2, of the 25,190 people originally in Method 1, Group 3, 69\% $(17,229)$ had no unobservable exposure time in the 30 days after discharge from all facility-based care, and those people were reassigned to Group 1; the remaining $31 \%(7,891)$ were readmitted to facilitybased care sometime during the 30-day exposure assessment period, and were reassigned to Group 2. Using Method 2, we increased the proportion of our sample with a full 30 days of observable time from $61 \%$ (Method 1) to $71 \%$ (Method 2). This difference is illustrated in Exhibit 3. Using the institutional discharge date definition (Method 2), patients in Group 1-those who were discharged to community and were observable all 30 days-were also younger, had fewer comorbidities, were less likely to be prevalent users of beta-blockers, had a shorter LOS, and were more likely to have survived 1-year post discharge than patients in Group 2
(Exhibit 5). However, the differences were less pronounced than the differences between groups constructed using Method 1.

On examination, using various thresholds for the number of observable days required to remain in the sample (Exhibit 6) demonstrates that it may be feasible to retain a significantly larger population than would be the case if those with unobservable time were censored. The characteristics of the subpopulation retained by allowing a greater amount of unobservable time include older age, more comorbidities, longer LOS, worse 1-year survival rate, and a slightly lower probability of being treated by beta-blockers as the threshold for the number of observable days decreased (Exhibit6). All groups created using the institutional discharge date, rather than the acute hospitalization discharge date, tended to be in worse health and yielded larger available populations for analysis. The mean and 
Exhibit 5. Frequencies and Means for Covariates for Institutional Discharge Date (Method 2) Groups

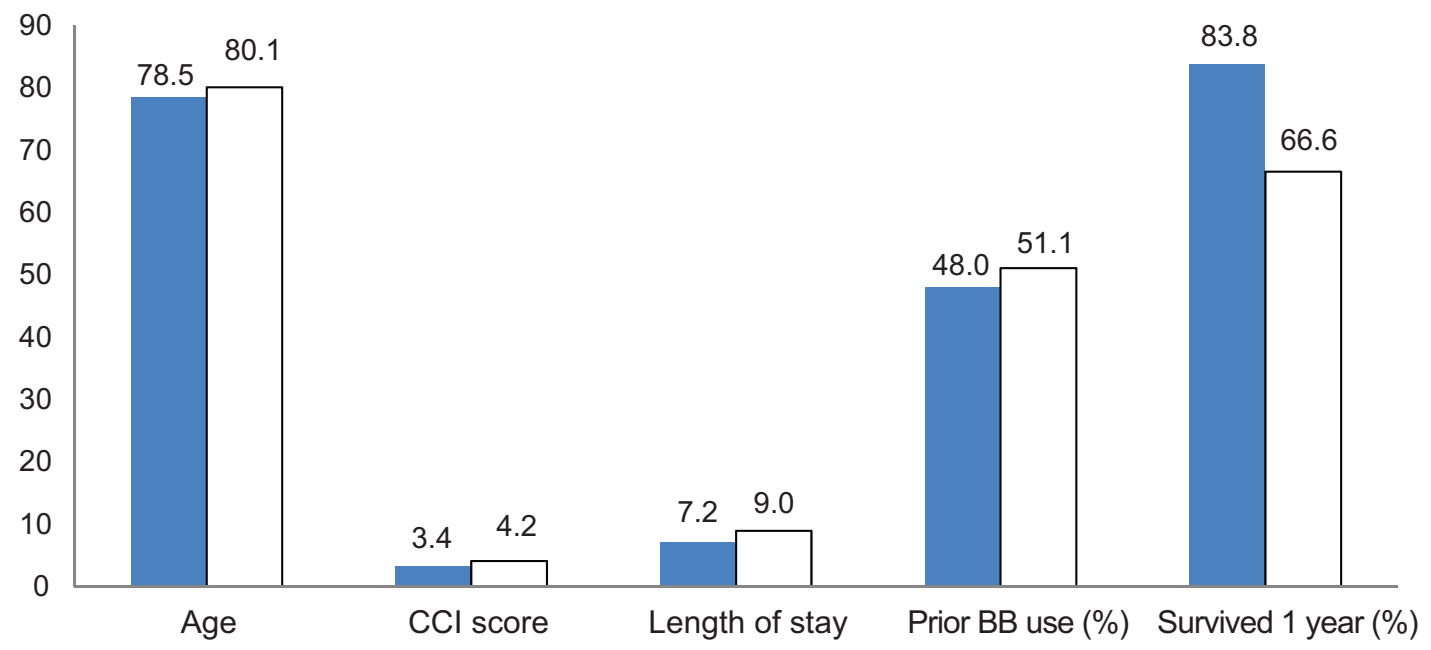

- Group 1 - discharged to community and observable all 30 days.

$\square$ Group 2 - discharged to community but were unobservable at some point during the first 30 days.

NOTE: All differences are significant at $\mathrm{p}<.0001$.

SOURCE: Calculations based on CCW Medicare AMI cohort 2007-2008, using enrollment and Part A and B fee-for-service claims, 2006-2009.

median time to first beta blocker prescription fill, for all groups and dates used, was well within the threshold number of days for inclusion in all cases, with the exception of the 10 day cut off.

Finally, the results of the logistic regression to predict beta blocker use in the 30 days post-acute care discharge (Method 1; see Exhibit 7) revealed that those discharged to institutional settings and immediately unobservable (Group 3) were significantly less likely to appear treated (adjusted OR $=0.5295 \%$ CI $0.51,0.54$ ), while those discharged to the community, but readmitted to facility-based care

Exhibit 6. Population, Proportion Treated, and Summary Statistics by Number of Observable Days Required to Remain in Sample

\begin{tabular}{|c|c|c|c|c|c|c|c|c|}
\hline \multirow[b]{2}{*}{ Observable days } & \multicolumn{4}{|c|}{ Acute discharge date } & \multicolumn{4}{|c|}{ Institutional discharge date } \\
\hline & 30 days & 20 days & 15 days & 10 days & 30 days & 20 days & 15 days & 10 days \\
\hline Sample size & 109,886 & 144,218 & 156,129 & 165,323 & 127,185 & 159,274 & 167,099 & 172,719 \\
\hline $\begin{array}{l}\text { Percent treated by beta } \\
\text { blockers }(\mathrm{BB})\end{array}$ & $70 \%$ & $68 \%$ & $67 \%$ & $66 \%$ & $67 \%$ & $66 \%$ & $66 \%$ & $65 \%$ \\
\hline $\begin{array}{l}\text { Mean(median) } \\
\text { days to first fill }\end{array}$ & $12.8(11)$ & $12.4(10)$ & $12.5(11)$ & $12.6(11)$ & $13.4(13)$ & $13.0(12)$ & $13.0(12)$ & $13.0(12)$ \\
\hline Mean age at index & 78.1 & 78.4 & 78.6 & 78.8 & 78.5 & 78.7 & 78.8 & 78.9 \\
\hline Mean CCI score & 3.2 & 3.4 & 3.4 & 3.5 & 3.4 & 3.5 & 3.5 & 3.6 \\
\hline Mean LOS & 6.6 & 7.0 & 7.2 & 7.4 & 7.2 & 7.4 & 7.5 & 7.6 \\
\hline Previous BB user & $48 \%$ & $49 \%$ & $49 \%$ & $49 \%$ & $48 \%$ & $49 \%$ & $49 \%$ & $49 \%$ \\
\hline Survived 1 year & $85 \%$ & $82 \%$ & $81 \%$ & $80 \%$ & $84 \%$ & $81 \%$ & $80 \%$ & $80 \%$ \\
\hline
\end{tabular}


Exhibit 7. Comparative Odds of Being Observed as Treated by Beta Blockers Using a 30-Day Observable Day Requirement or Adjustment for Total Number of Observable Days

\begin{tabular}{|c|c|c|c|c|c|c|}
\hline & $\begin{array}{r}\text { Met } \\
(\text { Acu } \\
\text { Discha }\end{array}$ & $\begin{array}{l}\text { d } 1 \\
\text { Care } \\
\text { Date) }\end{array}$ & $\begin{array}{r}\text { Me } \\
\text { (Inst } \\
\text { Discha }\end{array}$ & $\begin{array}{l}\text { d } 2 \\
\text { tional } \\
\text { e Date) }\end{array}$ & $\begin{array}{r}\text { Adjustmen } \\
\text { of Observal } \\
\text { Acute Dis }\end{array}$ & $\begin{array}{l}\text { or Number } \\
\text { Days using } \\
\text { arge Date }\end{array}$ \\
\hline & $\begin{array}{c}\text { Unadjusted } \\
\text { ORs } \\
(95 \% \mathrm{CL}) \\
\end{array}$ & $\begin{array}{c}\text { Adjusted } \\
\text { ORs } \\
(95 \% \mathrm{CL}) \\
\end{array}$ & $\begin{array}{c}\text { Unadjusted } \\
\text { OR } \\
(95 \% \text { CL }) \\
\end{array}$ & $\begin{array}{l}\text { Adjusted } \\
\text { ORs } \\
(95 \% \mathrm{CL})\end{array}$ & $\begin{array}{c}\text { Unadjusted } \\
\text { OR } \\
(95 \% \text { CL }) \\
\end{array}$ & $\begin{array}{l}\text { Adjusted } \\
\text { ORs } \\
(95 \% \mathrm{CL})\end{array}$ \\
\hline Group $1^{1}$ & 1.00 & 1.00 & 1.00 & 1.00 & NA & NA \\
\hline Group $2^{2}$ & $\begin{array}{c}0.60 \\
(0.59,0.62)\end{array}$ & $\begin{array}{c}0.71 \\
(0.70,0.73)\end{array}$ & $\begin{array}{c}0.59 \\
(0.57,0.60)\end{array}$ & $\begin{array}{c}0.70 \\
(0.68,0.71)\end{array}$ & NA & NA \\
\hline Group $3^{3}$ & $\begin{array}{c}0.38 \\
(0.37,0.39)\end{array}$ & $\begin{array}{c}0.52 \\
(0.51,0.54)\end{array}$ & NA & NA & NA & NA \\
\hline Age & $\begin{array}{c}0.97 \\
(0.97,0.97)\end{array}$ & $\begin{array}{c}0.97 \\
(0.97,0.97)\end{array}$ & $\begin{array}{c}0.97 \\
(0.97,0.97)\end{array}$ & $\begin{array}{c}0.97 \\
(0.97,0.97)\end{array}$ & $\begin{array}{c}0.97 \\
(0.97,0.97)\end{array}$ & $\begin{array}{c}0.97 \\
(0.97,0.97)\end{array}$ \\
\hline CCI score & $\begin{array}{c}0.86 \\
(0.86,0.86)\end{array}$ & $\begin{array}{c}0.89 \\
(0.89,0.90)\end{array}$ & $\begin{array}{c}0.86 \\
(0.86,0.87)\end{array}$ & $\begin{array}{c}0.89 \\
(0.89,0.89)\end{array}$ & $\begin{array}{c}0.86 \\
(0.86,0.86)\end{array}$ & $\begin{array}{c}0.90 \\
(0.90,0.91)\end{array}$ \\
\hline Inpatient LOS & $\begin{array}{c}0.96 \\
(0.96,0.96)\end{array}$ & $\begin{array}{c}0.98 \\
(0.98,0.98)\end{array}$ & $\begin{array}{c}0.96 \\
(0.96,0.96)\end{array}$ & $\begin{array}{c}0.98 \\
(0.97,0.98)\end{array}$ & $\begin{array}{c}0.96 \\
(0.96,0.96)\end{array}$ & $\begin{array}{c}0.99 \\
(0.99,0.99)\end{array}$ \\
\hline $\begin{array}{l}\text { Number of } \\
\text { observable days }\end{array}$ & NA & NA & NA & NA & $\begin{array}{c}1.06 \\
(1.06,1.06)\end{array}$ & $\begin{array}{c}1.05 \\
(1.05,1.05)\end{array}$ \\
\hline
\end{tabular}

with some unobservable time (Group 2), were slightly more likely to appear treated than those in Group 3, but still less likely to appear treated that those with no unobservable time (Group 1; adjusted $\mathrm{OR}=0.71$; 95\% CI 0.70,0.73). Exhibit 7 shows the corresponding adjusted odds ratio for those discharged to the community, but readmitted to facility-based care (Group 2) under Method 2, was 0.70 (95\% CI 0.68, 0.71). Under Method 1, adjustment for unobservable exposure time attenuated apparent relationships between age, comorbidity, acute LOS, and initiation of beta blockers. Results of the third regression, which replaced group membership with the number of observable days post-acute care discharge as a covariate, showed a strong positive relationship between observable days and the probability of appearing treated (adjusted $\mathrm{OR}=1.05$; 95\% CI 1.05,1.05).

\section{Discussion and Limitations}

In a cohort of 179,065 fee-for-service Medicare beneficiaries hospitalized for an AMI, who survived at least 30 days after discharge, we examined the extent and correlates of unobservable drug exposure time immediately following discharge, using two different methods of identifying the beginning of the treatment observation time frame. Some unobservable time in the 30 days after the acute care discharge occurred for $39 \%$ of patients; alternatively, using the institutional discharge date, $29 \%$ of patients had unobservable time. Regardless of the method used, patients who had unobservable time were generally older, had more comorbidities, had longer acute index stays, were less likely to have been taking beta blockers prior to admission, and had worse one-year survival 
rates. An exploration using various thresholds for the number of observable days required to remain in the analytic sample, or using the number of observable days as a covariate, demonstrated the feasibility of retaining larger cohort sizes to improve generalizability.

Comparative effectiveness research strives to be inclusive of routine practice settings. Sample exclusion criteria can potentially adversely impact this goal. Although a commonly used option for addressing unobservable medication exposure time is to exclude all patients with unobservable time in the period following the acute stay, nearly $40 \%$ of patients would be excluded in the present study. Because these are also the patients in poorer health, the study results will not generalize well to this important and large segment of elders. For this reason we examined two categories of unobservable exposure time: (1) those discharged to community care, but later readmitted to acute or other facilitybased care, and (2) those discharged immediately to post-acute facility-based care. Patients in both situations were much less likely to be classified as initiating beta blocking drugs compared to those who resided in the community all 30 days, although the difference was less pronounced for those discharged to home and readmitted later in the month. This likely reflects baseline health status, which we captured through proxy measures and which were worse for the group that was discharged immediately to post-acute care.

By using the discharge date from contiguous post-acute facility care as the index discharge date, 17,299 patients (10\% of the total cohort) who would previously have had unobservable time during the exposure assessment period no longer did. This subset had the lowest odds of being observed as initiating treatment, reinforcing the finding that potential bias from ignoring unobservable exposure time due to immediate post-acute facility care is more serious than that due to readmission during the exposure assessment time frame. Second, by using Method 2, which includes the index hospital stay plus immediate post-acute institutional care, $69 \%(17,299)$ of the least healthy patients from Group 3, Method 1 (immediately unobservable), were redistributed to Method 2, Group 1 (observable all 30 days). For investigators who choose to only retain this first group for inclusion in a study, significant gains in the generalizability of results have been made by measuring the exposure time frame using this method. For those who also choose to require a minimum number of observable days to remain in the sample or use the number of observable days as a covariate, this benefit is further realized.

There is clearly a tradeoff here between the accuracy of measuring drug exposure and external validity. Those whose exposure time is immediately unobservable due to discharge to a post-acute facility should be examined closely, because the longer the duration of facility-based care, the more likely that outcome events will occur that would not be captured in the numerator of the event rates. A more cautious approach may be to stratify on community vs. post-acute facility-based care during the exposure assessment time frame and examine the stratum-specific event rates.

The issue of whether to include or exclude patients with some unobservable time during the exposure assessment period, however, depends on the objectives of the study in question. If all patients with unobserved exposure time are excluded, selection bias creates estimates of the effect of treatment that are only generalizable to a population that is overwhelmingly healthier and more likely to survive in any case, whether medication is prescribed or not. However, if all patients are included, unobserved exposure time would need to be accounted for to avoid potentially misclassifying institutionally-treated patients as untreated. One approach is to use 
shorter overall exposure assessment time frames, which will reduce the number of patients who need to be excluded due to readmission in this interval. As we demonstrated in Exhibit 6, another approach is to decide upon a minimum number of observable Part $\mathrm{D}$ days in the exposure assessment period and exclude those who do not meet that threshold, or use the number of observable days as a covariate, which would enable an even larger and more representative group of patients to be retained in the sample. Empirical examination of the time to first fill, and a comparison of relevant covariates, can aid in determining an appropriate time period. For our sample, significant gains in sample size and potential generalizability were realized when relaxing the thirty day requirement. Combined with the use of an institutional discharge date as the beginning of the exposure assessment window, $96 \%$ of the original sample was usable by the time a 10 observation day threshold was reached, although this cut-off would be inadvisable to use, given that the mean time to first fill for beta blockers exceeded 10 days. In this example, requiring twenty days preserved $89 \%$ of the original sample; relaxing the requirement to fifteen days allowed for $93 \%$ retention.

For all of our analyses, we combined overlapping acute inpatient claims, and used the latest discharge date from the set of overlapping claims, to define the index dates for the AMI admission. We did this, because we assumed most analysts would be aware of this issue. However, it should be noted that failure to connect these claims would result in another source of unobservable exposure time if an investigator assumes the patient was discharged to community after the first claim. Similarly, because initiation of preventive therapies is not in keeping with hospice goals, we chose to eliminate hospice patients from our analysis rather than combining those claims into our institutional stay file. This also results in an understatement of the extent of unobservable exposure time in the population.

Our findings provide a case study for controlling for unobservable drug exposure time in the Medicare Part D population. We extend the findings from Suissa (2008), who demonstrated "immeasurable time" bias in many case-control studies that examined the impact of drug therapy on outcomes. He found that unobserved exposure time due to rehospitalization during the observation period leads, simultaneously, to an artificially lower probability of drug exposure, and a higher risk of death, among those with unobservable exposure time.

One of the limitations of our study is that we did not explore the impact of unobservable exposure time bias on measurement of medication dosage or adherence, which is arguably where such a bias might have the greatest effect due to the longer period of time under consideration. However, Caderette and Burden (2010) explored the misclassification that can arise when failing to take unobservable exposure time into account, and found that both persistence and compliance to osteoporosis drugs were underestimated as a result.

We used thirty days post-discharge to define treatment initiation when establishing our initial groups. Shorter periods would result in fewer exclusions due to unobservable time, but would classify some treated patients as untreated; for example, when failure to initiate therapy is identified and corrected at the first post-discharge physician visit. Any given study should examine the extent of this tradeoff and determine the treatment exposure period accordingly, as we did in Exhibit 7.

\section{Conclusion}

The extent of unobservable medication exposure time is considerable in the Medicare population 
and differentially affects individuals with worse health status. Comparative effectiveness studies strive to provide answers to questions for patients and physicians in routine care settings, and a great potential strength of administrative claims analyses is their inclusiveness of all patients, enabling the evaluation of treatment effectiveness in real world settings. This potential benefit is rapidly compromised when cohort exclusions are made. To retain patients with unobservable medication exposure time during the assessment period, we recommend examining the extent of observable days and time to first fill from the acute or institutional discharge date, stratifying on receipt of post-acute facility-based care, and when appropriate, using the discharge date from contiguous post-acute facility care as the index date for beginning the exposure assessment period. Ultimately, it may be desirable to evaluate treatment effects separately for these strata rather than, or prior to, excluding them entirely from the study.

\section{Correspondence}

Elizabeth A. Cook, M.S., Department of Pharmacy Practice and Science, University of Iowa College of Pharmacy, 115 S. Grand, Iowa City, IA 52242, Tel.319-384-2815, elizabeth-cook@uiowa.edu

\section{References}

Andrade, S. E., Kahler, K. H., Frech, F., \& Chan, K. A. (2006). Methods for evaluation of medication adherence and persistence using automated databases. Pharmacoepidemiology and Drug Safety, 15(8), 565-574. PubMed http://dx.doi. org/10.1002/pds. 1230

Cadarette, S. M., Solomon, D. H., Katz, J. N., Patrick, A. R., \& Brookhart, M. A. (2011). Adherence to osteoporosis drugs and fracture prevention: no evidence of healthy adherer bias in a frail cohort of seniors. Osteoporosis International, 22(3),
943-954. PubMed http://dx.doi.org/10.1007/ s00198-010-1309-Z

Cadarette, S. M., \& Burden, A. M. (2010). Measuring and improving adherence to osteoporosis pharmacotherapy. Current Opinion in Rheumatology, 22(4), 397-403. PubMed http:// dx.doi.org/10.1097/BOR.0b013e32833ac7fe

Cox, E., Martin, B. C., Van Staa, T., Garbe, E., Siebert, U., \& Johnson, D. L. (2009). Good Research Practices for Comparative Effectiveness Research: Approaches to Mitigate Bias and Confounding in the Design of Nonrandomized Studies of Treatment Effects Using Secondary Data Sources: The International Society for Pharmacoeconomics and Outcomes Research Good Research Practices for Retrospective Database Analysis Task Force Report-Part II. Value in Health, 12(8), 1053-1061.PubMed http:// dx.doi.org/10.1111/j.1524-4733.2009.00601.x

Dunn, O. J. (1961). Multiple Comparisons Among Means. Journal of the American Statistical Association, 56, 52-64. http://dx.doi.org/10.1080/ 01621459.1961 .10482090

Fitz-Simon, N., Bennett, K., \& Feely, J. (2005). A review of studies of adherence with antihypertensive drugs using prescription databases. [PubMed]. Therapeutics and Clinical Risk Management, 1(2), 93-106. PubMed http:// dx.doi.org/10.2147/tcrm.1.2.93.62915

Klabunde, C. N., Potosky, A. L., Legler, J. M., \& Warren, J. L. (2000). Development of a comorbidity index using physician claims data. Journal of Clinical Epidemiology, 53(12), 12581267. PubMed http://dx.doi.org/10.1016/S08954356(00)00256-0

Motheral, B., Brooks, J. M., Clark, M. A., Crown, W. H., Davey, P., Hutchins, D., . . Stang, P. A. (2003, March-April). Checklist for Retrospective 
Database Studies-Report of the ISPOR Task Force on Retrospective Databases. Value in Health, 6(2), 90-97. PubMed http://dx.doi. org/10.1046/j.1524-4733.2003.00242.x

Nikitovic, M., Solomon, D. H., \& Cadarette, S. M. (2010). Methods to examine the impact of compliance to osteoporosis pharmacotherapy on fracture risk: systematic review and recommendations. Therapeutic Advances in Chronic Disease, 1(4), 149-162. PubMed http:// dx.doi.org/10.1177/2040622310376137

Peterson, A. M., Nau, D. P., Cramer, J. A., Benner, J., Gwadry-Sridhar, F., \& Nichol, M. (2007). A Checklist for medication compliance and persistence studies using retrospective databases. Value in Health, 10(1), 3-12. PubMed http://dx.doi.org/10.1111/j.15244733.2006.00139.x

Ray, W. A. (2003). Evaluating medication effects outside of clinical trials: New-user designs. American Journal of Epidemiology, 158, 915920. PubMed http://dx.doi.org/10.1093/aje/ $\operatorname{kwg} 231$

Schneeweiss, S. (2007). Developments in postmarketing comparative effectiveness research. Clinical Pharmacology and Therapeutics, 82, 143-156. PubMed http://dx.doi.org/10.1038/ sj.clpt.6100249
Stukel, T. A., Fisher, E. S., Wennberg, D. E., Alter, D. A., Gottlieb, D. J., \& Vermeulen, M. J. (2007). Analysis of Observational Studies in the Presence of Treatment Selection Bias. Journal of the American Medical Association, 297(3), 278-285. PubMed http://dx.doi.org/10.1001/ jama.297.3.278

Sox, H. C., \& Greenfield, S. (2009). Comparative effectiveness research: a report from the Institute of Medicine. Annals of Internal Medicine, 151, 203-205. PubMed http://dx.doi.org/10.7326/ 0003-4819-151-3-200908040-00125

Suissa, S. (2008). Immeasurable time bias in observational studies of drug effects on mortality. American Journal of Epidemiology, 168(3), 329335. PubMed http://dx.doi.org/10.1093/aje/ kwn135

U.S. Department of Health and Human Services, Office of Inspector General (2009). Medicare Part D Payments for Beneficiaries in Part A Skilled Nursing Facility Stays in 2006 (OEI-02-07-00230). Washington, DC: Government Printing Office. Yang, Y., Thumula, V., Pace, P. F., Banahan, B. F., III, Wilkin, N. E., \& Lobb, W. B. (2009). Predictors of Medication Nonadherence Among Patients with Diabetes in Medicare Part D Programs: A Retrospective Cohort Study. Clinical Therapeutics, 31(10), 2178-2188. PubMedhttp:// dx.doi.org/10.1016/j.clinthera.2009.10.002 